\title{
La explotación sexual de niños, niñas y adolescentes durante el conflicto armado en Colombia ${ }^{1}$
}

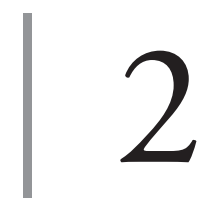

\author{
https://doi.org/10.21830/9789585284869.02
}

\author{
Jaime Cubides-Cárdenas ${ }^{2}$ \\ Andrea Carolina Paternina Feria ${ }^{3}$ \\ Paula Andrea Barreto Cifuentes ${ }^{4}$ \\ Universidad Católica de Colombia \\ Paola Alexandra Sierra-Zamora ${ }^{5}$ \\ Escuela Militar de Cadetes "General José María Córdova”
}

1 Este capítulo hace parte de los resultados del proyecto de investigación "Desafíos contemporáneos para la protección de derechos humanos en escenarios de posconflicto desde enfoques interdisciplinarios" del Grupo de Investigación Persona, Instituciones y Exigencias de Justicia, del Centro de Investigaciones Sociojurídicas, Facultad de Derecho, de la Universidad Católica de Colombia, registrado con el código COL0120899 de Minciencias. También contó con la colaboración del proyecto de investigación "La legitimidad de las Fuerzas Militares en la geopolítica nacional e internacional de Colombia" del Grupo de Investigación en Ciencias Militares, de la Escuela Militar de Cadetes "General José María Córdova” (ESMIC), registrado con el código COL0082556 de Minciencias. Los puntos de vista y los resultados de este artículo pertenecen a los autores y no reflejan necesariamente los de las instituciones participantes.

2 Abogado y especialista en Derecho Público de la Universidad Autónoma de Colombia. Magíster en Docencia e Investigación con énfasis en Ciencias Jurídicas de la Universidad Sergio Arboleda y magíster en Derecho de la misma casa de estudios. Estudiante del Doctorado en Derecho en la línea de investigación de Derecho Constitucional de la Universidad de Buenos Aires (Argentina). Docente asociado e investigador y líder del Grupo de Investigación Persona, Instituciones y Exigencias de Justicia, de la Universidad Católica de Colombia. Orcid: https://orcid.org/0000-0002-6542-6892 - Contacto: jacubides@ucatolica.edu.co

3 Auxiliar de investigación del Grupo de Investigación Persona, Instituciones y Exigencias de Justicia (Universidad Católica de Colombia) e integrante del semillero de investigación Observatorio de Justicia Constitucional y Derechos Humanos, vinculado al Centro de Investigaciones Sociojurídicas (CISJUC) de la Facultad de Derecho de la Universidad Católica de Colombia. Estudiante de la Facultad de Derecho de la misma casa de estudios. Orcid: https://orcid.org/0000-0002-7860-3069 - Contacto: acpaternina14@ucatolica.edu.co

4 Auxiliar de investigación del Grupo de Investigación Persona, Instituciones y Exigencias de Justicia (Universidad Católica de Colombia) e integrante del semillero de investigación Observatorio de Justicia Constitucional y Derechos Humanos, vinculado al Centro de Investigaciones Sociojurídicas (CISJUC) de la Facultad de Derecho de la Universidad Católica de Colombia. Estudiante de la Facultad de Derecho de la misma casa de estudios. Orcid: https://orcid.org/0000-0002-3863-0935 - Contacto: pabarreto04@ucatolica.edu.co

5 Abogada de la Universidad Católica de Colombia. Magíster en Derechos Humanos, Democracia y Justicia Internacional y estudiante del Doctorado en Derechos Humanos, Democracia y Justicia Internacional de la Universitat de València (España). Orcid: https://orcid.org/0000-0002-3146-7418 - Contacto: paola.sierra@esmic.edu.co 


\section{Introducción}

La explotación sexual comercial de niños ${ }^{6}$, niñas y adolescentes ${ }^{7}$ (en adelante, ESCNNA) en Colombia ha sido un grave problema que no tiene identificada una referencia temporal exacta, no obstante, durante décadas ha sido reconocida como un problema tangible en los últimos años (Cortés et al., 2011). En efecto, el registro histórico de este problema es una prioridad para el avance en su investigación y erradicación (Muñoz et al., 2016).

La explotación sexual comercial, en todo caso, es la continuación de situaciones y condiciones de vida en las que los derechos de esos NNA son vulnerados. Situaciones como el abandono, la expulsión de las escuelas y las carencias materiales y afectivas hacen que los menores queden expuestos a situaciones que los hacen especialmente propensos a caer en manos de quienes los someten al comercio sexual (Sosa, 2013, pp. 3-9).

Es importante destacar que, por tratarse de sujetos pasivos (los NNA), no puede descuidarse el tema de la inducción a la prostitución, que se define como "una conducta consistente en ejercer influencia psíquica en una persona para que esta adopte la decisión de practicar la prostitución por su cuenta y riesgo" (Mendoza, 2014, p. 79), lo cual es posible, debido al control que pueden tener los adultos frente a los menores de edad. Tal situación de indefensión de los NNA, en función de su condición de minoría de edad e inexperiencia de vida (ante una situación gravosa en cuanto a sus derechos), obliga al Estado a ejecutar acciones directas e inmediatas de tutela de sus derechos conforme a una obligación internacional detallada en el metaprincipio del interés superior del niño (Bermúdez-Tapia, 2012).

6 En el año 2009 se cumplieron 20 ańos de la declaración que da origen a la Convención Internacional sobre los Derechos del Nińo. A partir de una serie de encuentros se pretendía evaluar el estado de avance en las metas propuestas para las décadas de 1990 y del 2000, tanto en las regiones como en los países del mundo en los que se llevaron a cabo acciones en pro de la infancia.

7 "La explotación sexual comercial de niños, niñas y adolescentes (ESCNNA) es una violación fundamental a los derechos de la niñez. Abarca el abuso sexual por parte de un adulto y la remuneración en dinero o en especie para el nińo, niña, adolescente (NNA) o para una tercera persona o personas" (Davis, 2002). 


\section{Generalidades de la ESCNNA}

La expresión ESCNNA responde a un posicionamiento sobre el tema característico de la perspectiva de derechos basada en la Convención sobre los Derechos del Niño (Unicef, 2006/1989). De este modo se establece una diferencia radical entre prostitución (o trabajo sexual de personas adultas) y ESCNNA (Dilacio et al., 2012) ${ }^{8}$.

Para centrar el tema en materia judicial en Colombia, el delito de prostitución en menores de 18 años está penalizado en la Ley 599 de 2000 del Código Penal, y discrimina varios delitos referidos a la explotación sexual (capítulo IV): inducción a la prostitución (art. 213), proxenetismo con menor de edad (art. 213A), constreñimiento a la prostitución (art. 214), constreñimiento a la prostitución de menores (art. 217) y demanda de explotación sexual comercial de persona menor de 18 ańos (art. 217).

Del tema de la ESCNNA se desprende un grupo de problemáticas, catalogadas como modalidades según la Fundación Renacer (Fundación Renacer, s. f.) y adoptadas por el Instituto Colombiano de Bienestar Familiar (ICBF, 2014): utilización de niños, niñas y adolescentes en la prostitución; utilización de niños, niñas y adolescentes en la pornografía; el turismo sexual que vincula niños, niñas y adolescentes; la trata de niños, niñas y adolescentes con fines sexuales; los matrimonios serviles de niños, niñas y adolescentes; y la utilización de niños, niñas y adolescentes por grupos armados ilegales.

Desde el enfoque de los derechos humanos ${ }^{9}$, cuya importancia radica en que "una de las realidades que más aflige a la humanidad es la constante

8 Es importante tener presente en qué se fundamenta la diferencia entre la ESCNNA y la esclavitud, puesto que ambas son modos explotación laboral propias del capitalismo en las que la apropiación del explotador no se da sobre el producto del trabajo, sino sobre el propio cuerpo; no obstante, en el caso de la ESCNNA, también se da la apropiación sobre la sexualidad y el deseo de las víctimas. Si bien el trabajo infantil, en especial, en las llamadas peores formas, compromete el desarrollo pleno y genera consecuencias para la salud, en el caso de la ESCNNA, la cosificación de los niños y las secuelas psíquicas, físicas y sociales de estas experiencias adquieren un carácter devastador sobre sus posibilidades de desarrollo como personas (Dilacio et al., 2012).

9 El nuevo modelo de Estado ha introducido un concepto trascendental acerca de la preponderancia de la dignidad humana, alrededor de la cual giran los derechos humanos que son el sustento de esta misma (Cubides, 2013). A lo que cabe añadir que es una gran obligación del Estado evitar la afectación de los derechos de los NNA y que, cuando esto no sea posible, se le resarzan esos derechos fundamentales afectados durante la práctica del comercio sexual. Véase al respecto Cubides \& Barreto Cifuentes (2018). 
presencia de conductas por las cuales una persona en contra de otra, vulnera gravemente sus derechos fundamentales" (Cubides \& Barreto Cifuentes, 2018), y más aún en relación con los derechos de menores, se debe tener en cuenta que la víctima de ESCNNA no solamente es afectada en sus derechos a la integridad física y psicológica, en sus derechos sexuales y reproductivos, sino que además se ven vulnerados otro tipo de derechos como el de la salud, la educación, el derecho a una familia y a la recreación, entre otros. Esto, por supuesto, implica que los análisis que se han de realizar con respecto a esta problemática y las respuestas estatales, de la sociedad y de la familia deben considerar aspectos mucho más integrales y holísticos.

De igual manera, al abordar el tema de la ESCNNA como una violación a los derechos humanos de los niños, niñas y adolescentes, se parte de las responsabilidades de los diferentes actores en donde haya población infantil, pues es allí que existe la total obligación de garantizarles el pleno ejercicio de sus derechos (Cubides, Castro Buitrago, \& Barreto Cifuentes, 2017). Tal es el punto de partida para brindar recomendaciones a los gobiernos locales, teniendo en cuenta su obligación de respetar, proteger y hacer efectivos los derechos humanos, de forma que se diseñen y formulen políticas públicas, planes y programas enfocados en el desarrollo de la infancia que integren soluciones intersectoriales (ICBF, 2014)

\section{Evaluación de cifras referenciales de ESCNNA}

En este apartado se hace una verificación de cifras que arroja la ESCNNA en Colombia, cifras que ponen de manifiesto y permiten dimensionar el grado de vulneración de los derechos y la proporción de los delitos cometidos contra los niños, niñas y adolescentes. La figura 1 muestra el resultado del análisis descriptivo de las víctimas de explotación sexual comercial agregadas de acuerdo con los siguientes parámetros: i) explotación sexual con fines pornográficos, ii) explotación sexual con fines de prostitución y iii) otros delitos relacionados con la explotación sexual que ingresan al proceso administrativo de restablecimiento de derechos —PARD— (ICBF, 2014). 


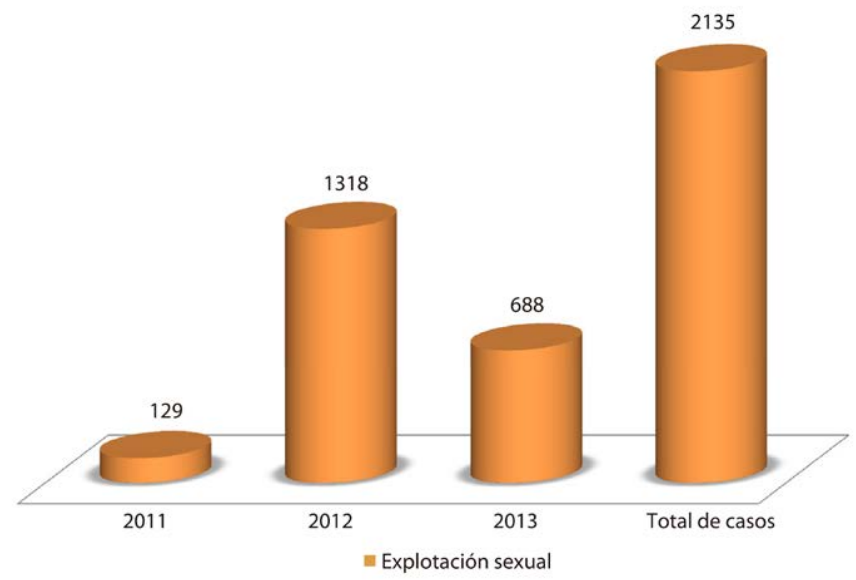

Figura 1. Número de casos que ingresan al PARD por el delito de explotación sexual comercial según el consolidado desde el 2011 hasta septiembre del 2013.

Fuente: ICBF (2014)

El total de casos que ingresan al PARD por explotación sexual comercial (cuya atención se inició con una acción administrativa o de otra naturaleza) son 2135, entre el 2011 y el 30 de septiembre del 2013. Esto representa el $1,94 \%$ del total de casos que ingresan al PARD (figura 1).

En dicho lapso se evidencia un notable crecimiento de ingresos al PARD por el delito de explotación sexual comercial en niños, niñas y adolescentes. El crecimiento porcentual más alto se presentó entre 2011 y 2012, con un $921,7 \%$, sin embargo, entre el 2012 y el 30 de septiembre del 2013 se presentó un decrecimiento del 47,8\% (figura 1).

Como se observa en la figura 2, para los tres primeros trimestres del 2012 se presentó un crecimiento importante de casos ingresados por el delito de explotación sexual comercial (967 casos) respecto al 2011, en cuyos tres primeros trimestres se registraron solo 77 casos. Sin embargo, para los tres primeros trimestres del 2013 se presentó una disminución de ingresos por este delito, con 688 casos.

Al realizar una comparación del cambio porcentual entre los tres trimestres del 2011, 2012 y 2013 para el delito de explotación sexual comercial en 
niños, niñas y adolescentes, se puede distinguir que para los primeros trimestres de 2011 y 2012 se evidencia un aumento de 1334,7\%, pasando de 23 casos en el 2011 a 330 casos en el 2012.

Por su parte, para el primer trimestre del 2012 y del 2013 se muestra una disminución porcentual del 25,7\%. Desde el segundo trimestre de 2011 hasta el 2012 se presentó un crecimiento porcentual de 1278,2\%, pasando de 23 casos en el 2011 a 317 casos en el 2012. Por su parte, para el mismo trimestre de 2012 a 2013 se muestra una disminución de 28,08\%. Para el tercer trimestre del 2011 hasta el 2012 el crecimiento porcentual fue de 961,2\%, en cambio, para el mismo trimestre del 2012 a 2013 se muestra una disminución del $34,65 \%$ (figura 2).

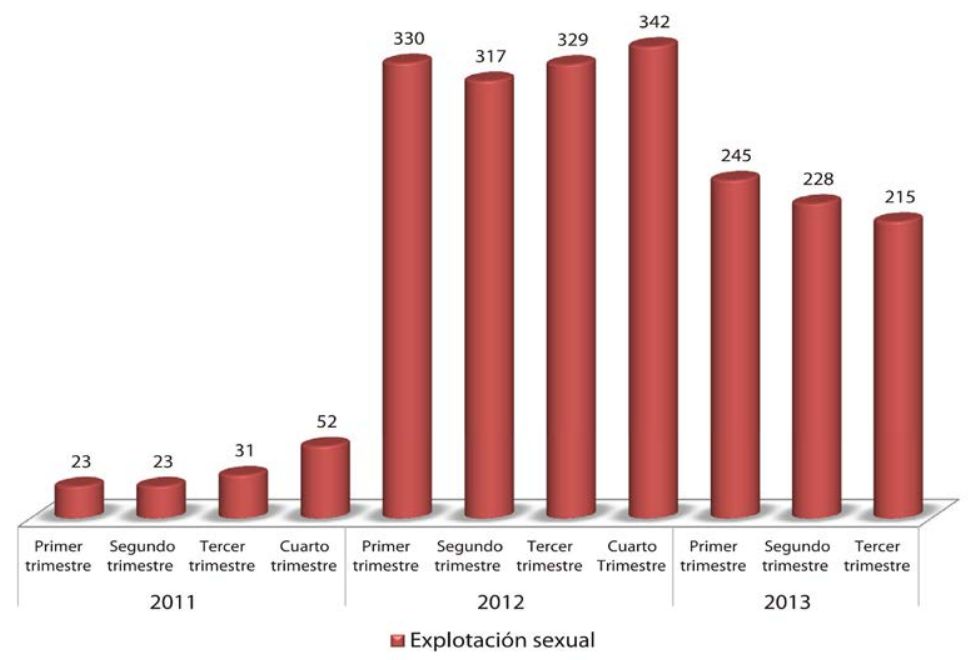

Figura 2. Número de casos que ingresan al PARD por el delito de explotación sexual comercial para los trimestres de 2011-2013, según motivo de ingreso.

Fuente: ICBF (2014)

El ingreso de nińos y adolescentes hombres para el primer y segundo trimestre del 2011 decreció en un 50\%, tendencia que no ocurrió para el segundo y cuarto trimestre de este mismo ańo: para el segundo y tercer trimestre del 2011 aumentó el número de casos en un 300\%, y para el tercer y cuarto trimestre aumentó en un $75 \%$ el número de ingresos, pasando de 4 reportes en el tercer trimestre a 7 registros para el cuarto trimestre. 
El comportamiento para el 2012 en las niñas y adolescentes mujeres fue similar al ocurrido para los trimestres del 2011 en los niños y adolescentes hombres, es decir, para el primer y segundo trimestre decreció el número de casos de niñas y adolescentes víctimas de la explotación sexual comercial en un $1,47 \%$, mientras que para el segundo y tercer trimestre aumentó en 1,86\%.

Por su parte, el tercer y cuarto trimestre de 2012 representó el crecimiento más importante del año con un 7,30\%, pasando de 274 casos en el tercer trimestre a 294 casos en el cuarto trimestre. En el caso de los niños y adolescentes hombres, entre el primer y el segundo trimestre de 2012 se presentó un decrecimiento del 15,79\%. Sin embargo, para el segundo y tercer trimestre, el comportamiento fue diferente porque hubo un incremento del cambio porcentual de un $14,58 \%$. Finalmente, para los dos últimos trimestres se da una disminución del 12,73\%, pasando de 55 ingresos a 48 en el cuarto trimestre del 2012 (figura 3) (ICBF, 2014).

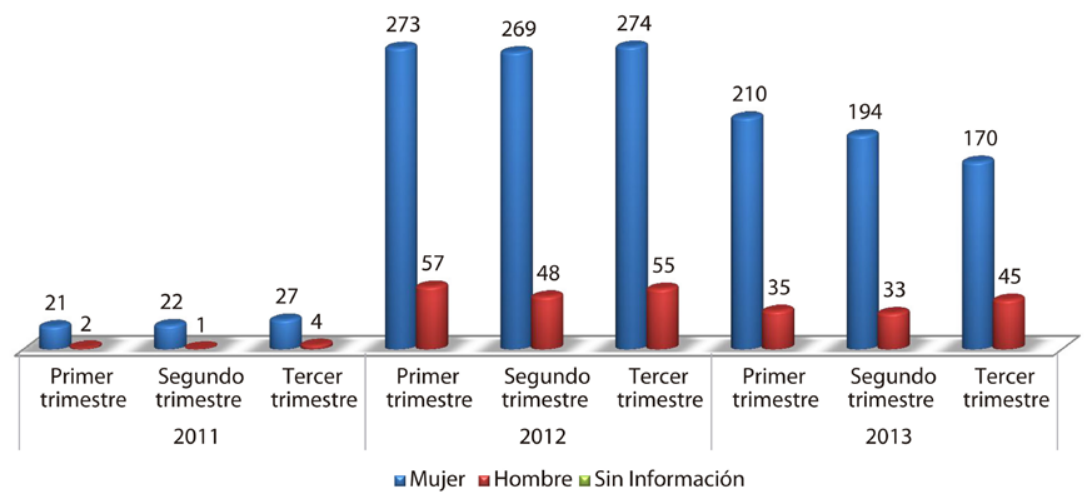

Figura 3. Número de casos que ingresan al PARD por el delito de explotación sexual comercial para los trimestres del 2011-2013, según sexo.

Fuente: ICBF (2014)

Además de toda la información brindada anteriormente, los datos de Unicef exponen que veinticinco mil menores son víctimas de la explotación sexual en Colombia, de los cuales dieciséis mil tienen entre 8 y 12 años de edad.

En el ámbito de los derechos humanos, los derechos de los niños y las niñas — que protegen a quienes son menores de dieciocho años- juegan un papel trascendental. La explotación sexual de menores de edad, bien sea 
comercial o no, da lugar a la contravención de lo establecido socioculturalmente y a la transgresión de varios derechos, lo da lugar a la tipificación de los delitos sexuales contra niños, niñas y adolescentes. Estos factores deben ser tenidos en cuenta por los Estados nación, las legislaciones, los operadores jurídicos y la sociedad en general, para trabajar en red de cultura protectora, que genere conciencia, comprensión y cambios significativamente positivos ante la crueldad, los orígenes y las consecuencias que desencadena este flagelo (Ossa-Estrada \& Muñoz-Echeverri, 2017).

\section{Conclusiones}

Es importante destacar la existencia de leyes, la constitucionalización de los derechos de los NNA y su protección especial (Bermúdez-Tapia, 2011), además de contar con diferentes instrumentos internacionales y los aportes y el esfuerzo de las fundaciones que existen en Colombia. Todo ello contribuye al trabajo que se realiza para la erradicación de la ESCNNA en Colombia, sin embargo, es imperante mencionar que, de acuerdo con las estadísticas presentadas en el presente capítulo, esta problemática se sigue presentando con altos grados de incidencia en la vida de los NNA en Colombia.

Una de las causas fundamentales de la ESCNNA es el desconocimiento parcial o total de las personas responsables de los NNA: sus padres o familiares. Esto provoca una situación de inseguridad jurídica, debido a que las leyes existentes y los mecanismos de protección que existen de los derechos de los NNA (comunidad que goza de especial protección constitucional) no son realmente efectivos.

\section{Referencias}

Bermúdez-Tapia, M. (2011). La constitucionalización del derecho de familia. Ediciones Caballero Bustamante

Bermúdez-Tapia, M. (2012). Derecho procesal de familia. Editorial San Marcos.

Cortés, R., Toro, E., \& Valdenegro, V. (2011). Estudio de las manifestaciones locales de explotación sexual comercial de niños y adolescentes, incluyendo la especificidad de victimas jóvenes hombres y los modelos de atención a victimas [Informe de investigación]. Repositorio de ECPAT International. http://www.ecpat.org/wp-content/uploads/legacy/Chile\%20Research.pdf 
Cubides, J. (2013). El origen del control de convencionalidad (CCV) y sus implicaciones para los Estados que reconocen el Sistema Interamericano de Derechos Humanos (SIDH). Ambiente Jurídico, 15, 103-125.

Cubides, J., \& Barreto Cifuentes, P. (2018). Análisis de las medidas cautelares en Colombia por parte del Sistema Interamericano de Derechos Humanos 2010-2015. En E. Velandia (Ed.), Tendencias contemporáneas del derecho procesal (pp. 389-420). Universidad Libre.

Cubides, J., Castro Buitrago, C. \& Barreto Cifuentes, P. (2017). El plazo razonable a la luz de los estándares de la jurisprudencia de la Corte Interamericana de Derechos Humanos. En J. Cubides (Ed.), Desafíos contemporáneos de la protección de los derechos humanos en el Sistema Interamericano (pp. 14-30). Universidad Católica de Colombia.

Davis, E. (2002). La explotación sexual comercial de niños, niñas y adolescentes en Panamá. IMUP.

Dilacio, G., Giorgi, V., \& Varela, C. (2012). Las representaciones sociales acerca de la Explotación Sexual Comercial de Niños, Niñas y Adolescentes y su relación con los paradigmas vigentes en el campo de la infancia y la adolescencia. Conocimiento y Sociedad, 2(1), 170-200. http://www.redalyc.org/html/4758/475847407008/

Fundación Renacer. (s. f.). Programas de Acción ESCNNA. https://fundacionrenacer.org/category/escnnal

Gómez, A. (2014). Prostitución de niñas y adolescentes: un acercamiento a su representación social en comerciantes de la merced. Península, 9(2), 131-152. https://doi.org/10.1016/ S1870-5766(14)71803-3

Instituto Colombiano de Bienestar Familiar. (2014). Análisis de la situación de explotación sexual comercial en Colombia, una oportunidad para garantizar la protección de niños, niñas y adolescentes. http://www.derechoshumanos.gov.co/Observatorio/Publicaciones/ Documents/2014/140718_libro_explotacion_sexual_nna.pdf

Mendoza, J. F. (2014). ¿Debe ser punible la inducción a la prostitución? Estudio de su fundamentación, estructura dogmática y problemática. Novum Jus, 8(1), 77-93. http://dx.doi. org/10.14718/NovumJus.2014.8.1.4

Muñoz, I., Noreña, C., Rodríguez, S., Ossa, D., \& Bastidas, D. (2016). Escenarios de explotación sexual comercial de niños, niñas y adolescentes, modalidad abierta, Medellín, 2012. Revista Facultad Nacional de Salud Pública, 34(1), 9-19. https://doi.org/10.17533/udea. rfnsp.v34n1a02

Ossa-Estrada, D. A., \& Muñoz-Echeverri, I. F. (2017). Explotación sexual comercial de niños, niñas y adolescentes: significados y prácticas de trabajadores/as y residentes del centro de Medellín (Colombia), 2015. Salud Colectiva, 13(1), 19-34. https://doi.org/10.18294/ sc. 2017.1107

Sosa, P. (2013). Explotación sexual comercial de niños, niñas y adolescentes: la construcción social del problema. Cambios y continuidades en Uruguay [Tesis de licenciatura, Universidad de la República]. Repositorio Colibrí de la Universidad de la República. https://www.colibri. udelar.edu.uy/jspui/bitstream/123456789/7235/1/TTS_SosaPierina.pdf

Unicef. (2006/1989). Convención sobre los Derechos del Niño. 